\title{
On the Impact of Imperfect Cophasing in Uncoded and LDPC-Coded EGC Receivers over Generalized Fading Channels
}

\author{
Goran T. Djordjevic ${ }^{1}$, Ivan B. Djordjevic ${ }^{2}$, George K. Karagiannidis ${ }^{3}$ \\ ${ }^{1}$ Telecommunications Department, Faculty of Electronic Engineering, University of Nis \\ Nis 18000, Serbia, e-mail: goran@elfak.ni.ac.yu \\ ${ }^{2}$ University of Arizona, Department of Electrical and Computer Engineering \\ 1230 E. Speedway Blvd., Tucson, AZ 85721, USA, e-mail: ivan@ece.arizona.edu \\ ${ }^{3}$ Aristotle University of Thessaloniki, Department of Electrical \& Computer Engineering \\ Thessaloniki 54 124, Greece, e-mail: geokarag@auth.gr
}

\begin{abstract}
We study the performance of unbalanced partially coherent uncoded and low-density parity-check (LDPC)-coded equal gain combining (EGC) receiver operating over generalized $\alpha-\mu$ fading channels, when the binary phase-shift keying (BPSK) and quaternary phase-shift keying (QPSK) are used. We determine the bit-error ratio (BER) performance degradation due to the imperfect reference signal recovery, receiver unbalancing and fading. Furthermore, we design large girth quasi-cyclic LDPC code with high code rate, suitable for use in communications over generalized fading channels. The proposed LDPC code does not exhibit the error floor phenomena, in the region of interest, even in the presence of imperfect cophasing and receiver unbalances.
\end{abstract}

Keywords - Bit error rate, equal gain combining, low-density parity-check (LDPC) codes, phase error, phase-shift keying

\section{INTRODUCTION}

In order to combat the effects of multipath fading in wireless communication systems, the concept of spatially separated antennas at the receiver is used. The multiple versions of the same information-bearing signal picked up by these antennas are combined appropriately to increase the overall instantaneous signal-to-noise ratio (SNR). The widely used diversity techniques are selection combining (SC), switch and stay combining (SSC), equal gain combining (EGC) and maximum ratio combining (MRC). The EGC is a suboptimal combining technique achieving slightly smaller values of the instantaneous SNR than MRC, but on the other hand it has lower implementation complexity than that of MRC because it does not require estimation of the fading envelopes. According to the EGC technique, the received signals at all branches are cophased, equally weighted and summed to give the resultant output signal. The estimation of the received signal phase, required for cophasing, is accomplished by using a modulated or unmodulated carrier [1].

Previous papers concerning EGC diversity [2]-[5] (and references therein) assumed the received signal phase estimation to be perfect. Only [6], [7] considered the influence of the imperfect estimation of the received signal phase on the system performance. The authors in [6] discussed the phase error influence on the bit error rate (BER) values in detecting binary phase-shift keying (BPSK) and quaternary phase-shift keying (QPSK) signals. The analysis was done under the assumption of identical and statistically independent Rayleigh fading. In [7] the authors derived closed-form expressions for the outage probability and average BER in detecting BPSK and QPSK signals transmitted over correlated Nakagami- $m$ fading channels. The EGC technique with dual branches was also observed in that paper.

This paper studies the performance of unbalanced EGC receivers with imperfect cophasing, operating over nonidentical generalized $\alpha-\mu$ (or generalized Gamma) fading channels [8], [9]. The difference between the incoming and the estimated signal phase is a stochastic process which is assumed to follow the Tikhonov distribution, as in [6], [7], [10]. Notice that the Nakagami- $m$, exponential, Weibull, one-sided Gaussian and Rayleigh distribution that are used for modeling multipath fading channels, as well as Gamma and Lognormal (as a limiting case) distributions that are used for modeling shadow fading are special instances of the generalized $\alpha-\mu$ distribution [8], [9]. Despite the fact that the $\alpha-\mu$ distribution includes several widely used fading distributions as special cases, only two papers [4], [5] considered the performance of EGC receiver in $\alpha-\mu$ fading channel, and analysis in both papers was done under assumption of perfect cophasing. Unlike previously mentioned papers [2]-[7], where the uncoded signal detection was observed, in this paper we determine the average BER for both uncoded and low-density parity-check (LDPC)-coded BPSK and QPSK signals. To facilitate the implementation at high-speeds the LDPC codes are designed in quasi-cyclic fashion. To avoid the error floor phenomena for transmission over the generalized fading channels in the presence of imperfect phase recovery and receiver unbalances the LDPC codes of large girth are employed.

The rest of the paper is organized as follows. Section II describes the system model, the LDPC code design, and the description of evaluation of symbol and bit reliabilities. The numerical results, followed by discussions, are presented in Section III. Section IV contains the summary of the main results and provides some concluding remarks. 


\section{SYSTEM AND CHANNEL MODEL}

\section{A. Channel model}

According to the generalized fading channel model, at a certain given point the received signal is a sum of $n$ multipath components, and the propagation channel is such that the resulting signal envelope is a nonlinear function of the modulus of the sum of the components. Supposing that this nonlinearity is in the form of a power parameter $\alpha_{i}>0$, the generalized $\alpha-\mu$ fading envelope at the $i$-th receiver branch (Fig. 1) can be presented as [9]

$$
r_{i}^{\alpha_{i}}(t)=\sum_{j=1}^{n}\left[x_{F i j}^{2}(t)+y_{F i j}^{2}(t)\right]
$$

where $x_{F i j}(t)$ and $y_{F i j}(t)$ are the zero-mean mutually independent Gaussian processes with identical variances $\operatorname{Var}\left\{x_{F i j}(t)\right\}=\operatorname{Var}\left\{y_{F i j}(t)\right\}=\sigma_{F i}{ }^{2}$, with $\operatorname{Var}\{$.$\} denoting variance$ operator. The corresponding $\alpha-\mu$ probability density function (PDF) is given by [8], [9]

$$
p_{r_{i}}\left(r_{i}\right)=\frac{\alpha_{i} \mu_{i}^{\mu_{i}} r_{i}^{\alpha_{i} \mu_{i}-1}}{\hat{r}_{i}^{\alpha_{i} \mu_{i}} \Gamma\left(\mu_{i}\right)} \exp \left(\mu_{i} \frac{r_{i}^{\alpha_{i}}}{\hat{r}_{i}^{\alpha_{i}}}\right),
$$

where $\Gamma($.$) is the Gamma function [11, eq. (8.310/1)], and \mu_{i}>0$ is the inverse of the normalized variance of $r_{i}^{\alpha_{i}}$

$$
\mu_{i}=\mathrm{E}^{2}\left\{r_{i}^{\alpha_{i}}\right\} / \operatorname{Var}\left\{r_{i}^{\alpha_{i}}\right\}
$$

where $\mathrm{E}\{$.$\} is the expectation operator, and \hat{r}_{i}$ is a $\alpha_{i}$-root mean value

$$
\hat{r}_{i}=\sqrt[\alpha_{i}]{E\left\{r_{i}^{\alpha_{i}}\right\}}
$$

\section{B. Receiver model}

The signal at the $i$-th receiver antenna (see Fig. 1) can be written as

$$
s_{i}(t)=r_{i}(t) e^{j \gamma_{i}(t)} A e^{j\left(\omega_{0} t+\phi_{n}\right)}+n_{i}(t), \quad i=1,2, \ldots, L,
$$

where $r_{i}(t)$ is the fading envelope, $\gamma_{i}(t)$ is the random phase shift occurred during signal transmission over a fading channel. The fading at each antenna is frequency nonselective, it does not change for the duration of symbol (slow fading), it is independent from symbol to symbol and there is no correlation between fading on different antennas. The amplitude of useful signal is denoted with $A$ and it can be assumed without loss of generality that it is equal to one. With $\phi_{n}$ we denote the signal phase in which information about the symbol is imposed. In the case of the BPSK signal $\phi_{n}$ has one of the following values: $\{0, \pi\}$, and in the case of the QPSK signal $\phi_{n}$ has one of the following values: $\{\pi / 4,3 \pi / 4,5 \pi / 4$, $7 \pi / 4\}$. The zero mean Gaussian noise with variance $\sigma_{i}^{2}$ at the $i$ th receiver branch is denoted with $n_{i}(t)$. The standard deviation of this Gaussian noise is given by

$$
\sigma_{i}=\sqrt{\mathrm{E}\left\{r_{i}^{2}\right\} /\left[2 R \log _{2}(M) 10^{\gamma_{b 1} / 10} \exp (-\delta(i-1))\right]},
$$

where $R$ is the code rate (for uncoded signal $R=1$ ), $M$ is the number of phase levels, $\gamma_{b 1}$ is the average signal-to-noise ratio per information bit for the first receiver branch and is given in decibels, and $\delta$ is the receiver unbalance parameter. The receiver operates with the exponentially decaying power delay profile.

After signal cophasing at all branches, the resulting signal after combining is

$$
z(t)=\sum_{i=1}^{L}\left[A r_{i}(t) e^{j \phi_{n}} e^{j \varphi_{i}(t)}+n_{i}(t)\right],
$$

where $L$ is the number of the receiver branches. The difference between the receiver signal phase $\gamma_{i}(t)$ at the $i$-th receiver branch and the estimated phase $\hat{\gamma}_{i}(t)$ at that receiver branch is denoted with $\varphi_{i}(t)=\gamma_{i}(t)-\hat{\gamma}_{i}(t)$. If the phase estimation is done from unmodulated carrier by using phase-locked loop (PLL) and if only the Gaussian noise is present in the phaselocked loop circuit, then the PDF of this phase error is [6], [7], [10]

$$
p_{\varphi_{i}}\left(\varphi_{i}\right)=\frac{1}{2 \pi} \frac{\exp \left(\varsigma_{i} \cdot \cos \left(\varphi_{i}\right)\right)}{\mathrm{I}_{0}\left(\varsigma_{i}\right)}, \quad-\pi<\varphi_{i} \leq \pi,
$$

where $\mathrm{I}_{0}(x)$ is the modified Bessel function of the first kind and zero order for the argument $x\left[11\right.$, eq. (8.406)], $\zeta_{i}$ is the signalto-noise ratio in the PLL circuit at the $i$-th receiver branch, which is related to the phase error variance $\sigma_{\varphi}^{2}[6],[7],[10]$ by

$$
\zeta_{i}=1 / \sigma_{\varphi_{i}}^{2}
$$

The BER of uncoded BPSK and QPSK signal detection, assuming EGC receiver, are respectively given by

$$
B E R=\frac{1}{2} \iint_{\mathbf{r} \varphi} \operatorname{erfc}\left(\frac{\sum_{i=1}^{L} r_{i} \cos \varphi_{i}}{\sqrt{2} \sigma}\right) p_{\varphi}(\varphi) p_{\mathbf{r}}(\mathbf{r}) d \varphi d \mathbf{r}
$$

and

$$
\begin{gathered}
B E R=0.25 \iint_{\mathbf{r}}\left\{\operatorname{erfc}\left(\frac{\sum_{i=1}^{L} r_{i} \cos \left(\pi / 4-\varphi_{i}\right)}{\sqrt{2} \sigma}\right)+\right. \\
\left.+\operatorname{erfc}\left(\frac{\sum_{i=1}^{L} r_{i} \cos \left(\pi / 4+\varphi_{i}\right)}{\sqrt{2} \sigma}\right)\right\} p_{\varphi}(\varphi) p_{\mathbf{r}}(\mathbf{r}) d \varphi d \mathbf{r},
\end{gathered}
$$

where

$$
\sigma=\sqrt{{\sigma_{1}{ }^{2}+\sigma_{2}{ }^{2}+\ldots+{\sigma_{L}}^{2}}}
$$


$\operatorname{erfc}($.$) is the complementary error function [11, eq. (7.1.2.)],$ $p_{\varphi}(\varphi)$ is the joint probability density function of the vector $\varphi=\left(\varphi_{1}, \varphi_{2, \ldots} \varphi_{L}\right)$, which is, considering that $\varphi_{i}, i=1,2, \ldots L$, are independent, given by

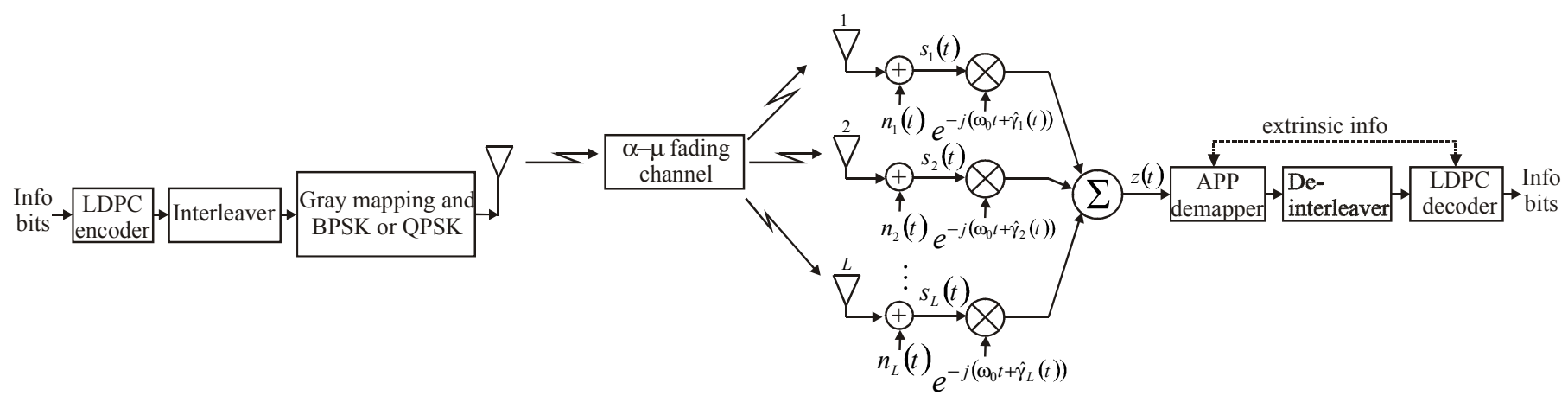

Fig. 1. System model

$$
p_{\varphi}(\varphi)=\prod_{i=1}^{L} p_{\varphi_{i}}\left(\varphi_{i}\right)
$$

and $p_{\mathbf{r}}(\mathbf{r})$ is the joint probability density function of the vector $\mathbf{r}=\left(r_{1}, r_{2}, \ldots, r_{L}\right)$, which is, considering that also $r_{i}, i=1,2, \ldots L$, are independent, given by

$$
p_{\mathrm{r}}(\mathrm{r})=\prod_{i=1}^{L} p_{r_{i}}\left(r_{i}\right) .
$$

\section{Encoding and decoding algorithm}

The LDPC codes under study in this paper belong to the class of quasi-cyclic (QC) LDPC codes [12], [13]. The QC codes, lead to encoder that can be implemented based on shiftregisters and modulo-2 adders, while complexity of decoder is low compared to random LDPC codes. The parity-check matrix of LDPC codes considered here can be written in the following form

$$
H=\left[\begin{array}{ccccc}
I & I & I & \ldots & I \\
I & P^{S[1]} & P^{S[2]} & \ldots & P^{S[c-1]} \\
I & P^{2 S[1]} & P^{2 S[2]} & \ldots & P^{2 S[c-1]} \\
\ldots & \ldots & \ldots & \ldots & \ldots \\
I & P^{(v-1) S[1]} & P^{(v-1) S[2]} & \ldots & P^{(v-1) S[c-1]}
\end{array}\right],
$$

where $I$ is $p \times p$ ( $p$ is a prime number) identity matrix, $P$ is $p \times p$ permutation matrix $\left(p_{i, i+1}=p_{p, 1}=1, i=1,2, \ldots, p\right.$-1; other elements of $P$ are zeros), while $v$ and $c$ represent the number of rows and columns in (15), respectively. The set of integers $S$ are to be carefully chosen from the set $\{0,1, \ldots, p-1\}$ so that the cycles of short length, in corresponding Tanner graph representation of (15) are avoided. The minimum distance for LDPC code is given by the Tanner bound [14]:

$$
d \geq\left\{\begin{array}{c}
1+\frac{v}{v-2}\left((v-1)^{\lfloor(g-2) / 4\rfloor}-1\right), \quad g / 2=2 m+1 \\
1+\frac{v}{v-2}\left((v-1)^{\lfloor(g-2) / 4\rfloor}-1\right)+(v-1)^{\lfloor(g-2) / 4\rfloor}, \quad g / 2=2 m
\end{array}\right.
$$

where $g$ and $v$ are respectively the girth of the LDPC code and the column weight of the parity check matrix. The operator \lfloor\rfloor indicates the largest integer that is smaller or equal to the enclosed number. Equation (16) shows that the linear increase in the girth results in exponentially increase in the minimum distance. Notice that this bound is tight only for short codes (in the order of hundreds), nevertheless it provides a guideline how to design the LDPC codes of large minimum distance. Moreover, the use of large girth LDPC de-correlates the extrinsic information in decoding process.

Example: By selecting $p=673$ and $S=\{0,2,5,13,20,37$, $58,91,135,160,220,525\}$ an LDPC code of rate 0.75 , girth $g=10$, column weight 3 and length $N=8076$ is obtained.

The decoding algorithm, as mentioned above, is based on sum-product with correction term algorithm [15]. The complexity of this algorithm is low and suitable for fieldprogrammable gate array (FPGA) or very-large-scale integration (VLSI) implementation.

\section{Symbol and bit log-likelihood ratios calculation}

The samples of in-phase and quadrature channels are forwarded to the a posteriori probability (APP) demapper, which determines the symbol log-likelihood ratios (LLRs) $\lambda(s)$ by

$$
\begin{aligned}
& \lambda(s)=-\frac{\left\{z_{I}-\operatorname{Re}[\operatorname{MPSK}(\operatorname{map}(s))]\right\}^{2}}{2 c^{2}}- \\
& -\frac{\left\{z_{Q}-\operatorname{Im}[\operatorname{MPSK}(\operatorname{map}(s))]\right\}^{2}}{2 c^{2}} ; \quad s=1,2, \ldots, 2^{n_{b}}
\end{aligned}
$$

where $z_{I}$ and $z_{Q}$ denote the sample of in-phase and quadrature channel outputs (see Eq. (7)), MPSK denotes the MPSK- 
constellation diagram, $\sigma^{2}$ denotes the variance of the Gaussian noise process, and $\operatorname{map}(s)$ denotes a corresponding mapping rule (Gray mapping is applied here). ( $n_{b}$ denotes the number of bits per symbol.) Let us denote by $b_{j}$, the $j$ th bit in an observed symbol $s$ binary representation $\boldsymbol{b}=\left(b_{1}, b_{2}, \ldots, b_{n \mathrm{~b}}\right)$. The bit LLRs required for LDPC decoding are calculated from symbol LLRs by

$$
L\left(b_{j}\right)=\log \frac{\sum_{s: b_{j}=0} \exp (\lambda(s))}{\sum_{s: b_{j}=1} \exp (\lambda(s))} .
$$

Therefore, the $j$ th bit reliability is calculated as the logarithm of the ratio of a probability that $b_{j}=0$ and probability that $b_{j}=1$. In the nominator, the summation is done over all symbols $s$ having 0 at the position $j$, while in the denominator over all symbols $s$ having 1 at the position $j$. The extrinsic LLRs are iterated backward and forward until convergence or predetermined number of iterations has been reached, as illustrated in Fig. 1 by dashed arrows.

\section{NUMERICAL RESULTS}

The assumption is that in all receiver branches the phase noise standard deviation is the same: $\sigma_{\varphi 1}=\sigma_{\varphi^{2}}=\ldots=\sigma_{\varphi L}=\sigma_{\varphi}$, and the fading parameters are also the same: $\alpha_{1}=\alpha_{2}=\ldots=\alpha_{L}=\alpha$, $\mu_{1}=\mu_{2}=\ldots=\mu_{L}=\mu$. For uncoded signal detection we present both numerical results obtained by numerical integration and those obtained by Monte Carlo simulations, and as it is evident there is excellent agreement.

The numerical integration in (10) and (11) was performed by using Gaussian quadrature formulas with previously given precision accuracy.

The simulation model can be summarized as follows. Information bits are uniformly generated and fed into LDPC encoder with encoding process briefly described in the preceding section. The LDPC encoder output bits are Gray mapped and give the information bearing phase $\phi_{n}$. The fading envelope $r_{i}(t)$ is generated by using (1). The phase error $\varphi_{i}(t)$ with the Tikhonov PDF is generated by the acceptance/rejection method [16, p. 381-382]. The $\sigma_{\phi^{i}}$ is needed for generating the phase error ((8) and (9)). The Gaussian noise $n_{i}(t)$, from (7), with zero mean and standard deviation given by (6), is generated by using the algorithm from [17]. The samples of $z(t)$, given by (7), are fed to the APP demapper, deinterleaver and LDPC decoder that is described in the preceding section. The simulation results are obtained for 25 iterations in sum-product algorithm. The system model from Fig. 1 incorporates block interleaver and deinterleaver with sufficiently dimension in order to insure the fading samples between two successive symbols are uncorrelated. The numerical results are obtained by applying Monte Carlo simulations. The BER values are estimated on the basis of 3000 information bit errors. In addition, minimum number of information words (consisting of 6059 information bits) that is used during evaluation of any BER value is 200 .
Figs. 2 and 3 present the influence of imperfect reference signal phase recovery on BER values in detecting uncoded BPSK and QPSK signals. The QPSK modulation format is much more sensitive to the phase error than the BPSK modulation format. In the case of uncoded BPSK, the BER values are constant up to about $\sigma_{\varphi}=16^{0}$, while for uncoded QPSK, BER values start rising always for $\sigma_{\varphi}=8^{0}$.

The influence of EGC receiver unbalance on BER values in detecting uncoded QPSK signals is illustrated in Fig. 4. The unbalance parameter $\delta$ considerably influence the BER values in the range of moderate $\gamma_{b 1}$ values. For $\sigma_{\varphi}=12.5^{\circ}$, in order to achieve $\mathrm{BER}=10^{-5}$ it is necessary to paid $\gamma_{b 1}$ penalty of about $2.8 \mathrm{~dB}$ if $\delta$ is changed from ideal case to $\delta=1$. However, in the regime of high values of $\gamma_{b 1}$, the parameter $\delta$ does not influence the BER values that are predominantly influenced by phase noise standard deviation. For $\sigma_{\varphi}=12.5^{\circ}$ all BER dependences tend to BER floor of about $10^{-6}$ regardless the amount of EGC receiver unbalance.

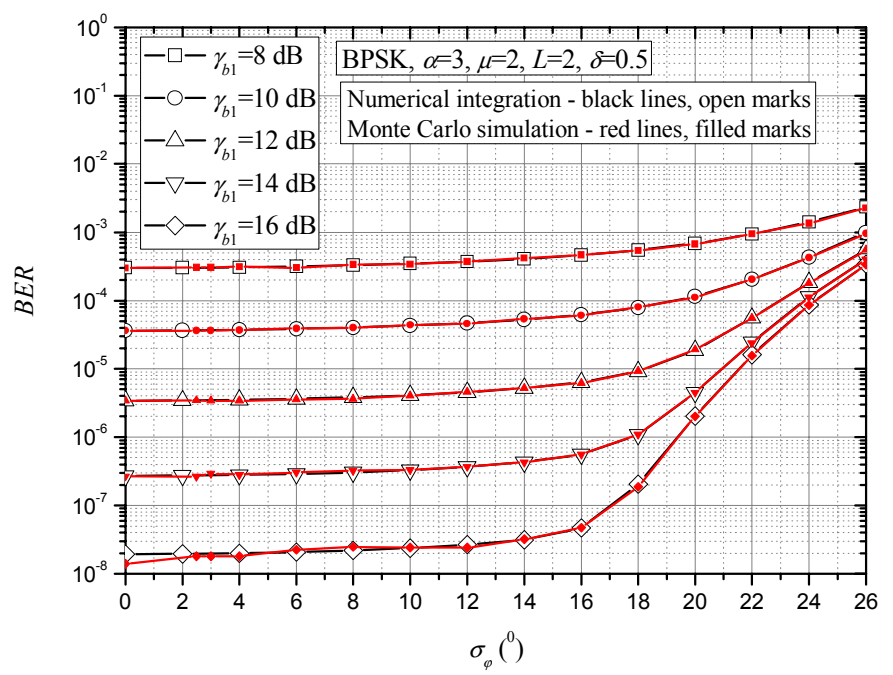

Fig. 2. BER of uncoded BPSK as a function of phase error standard deviation

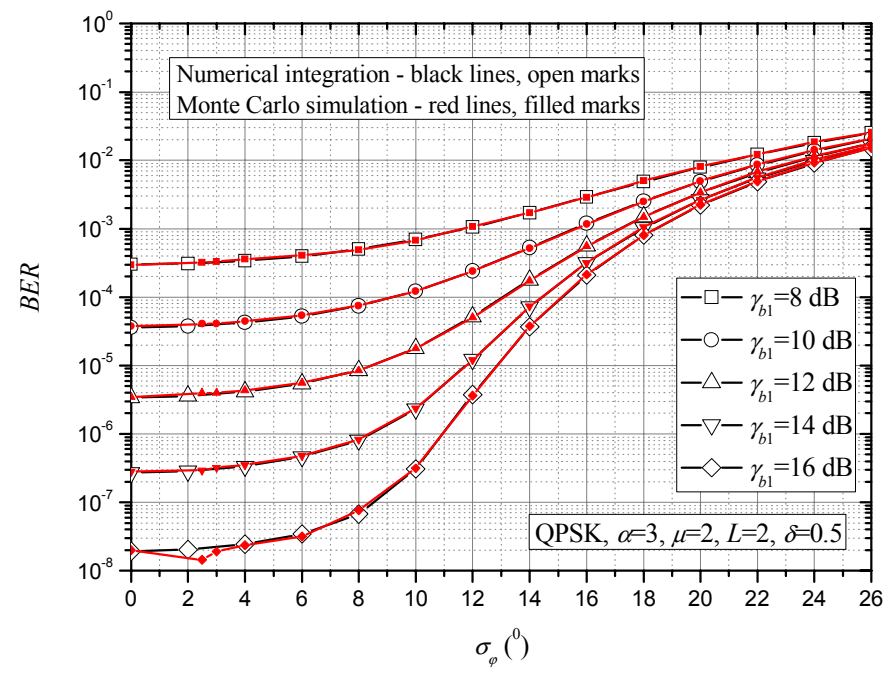

Fig. 3. BER of uncoded QPSK as a function of phase error standard deviation 
Fig. 5 shows the influence of phase error deviation on the average BER values of uncoded and LDPC-coded QPSK signal detection. In the case of uncoded QPSK, the random phase error causes appearance of the BER floor. The values of the phase error standard deviation considerably affect the values of this BER floor. For $\sigma_{\varphi}=20^{\circ}$, the BER floor value is $10^{3}$ times greater then for $\sigma_{\varphi}=12.5^{0}$. It is evident that the LDPC code $(8076,6059)$ with high code rate $R=0.75$ has good performance under considered influences. For BER of $10^{-5}$, in the cases of $\sigma_{\varphi}=0^{0}$ and $\sigma_{\varphi}=12.5^{\circ}$ the corresponding coding gains are $9.4 \mathrm{~dB}$ and $13 \mathrm{~dB}$, respectively. That means that this LDPC code performs very well not only in the generalized fading channel under the influence of unbalancing, but it is also very tolerant to the imperfect cophasing. The higher the value of phase noise standard deviation, the larger the coding gain value is. Notice that this code does not exhibit the BER floor phenomena down to $10^{-6}$ even for $\sigma_{\varphi}=20^{0}$.

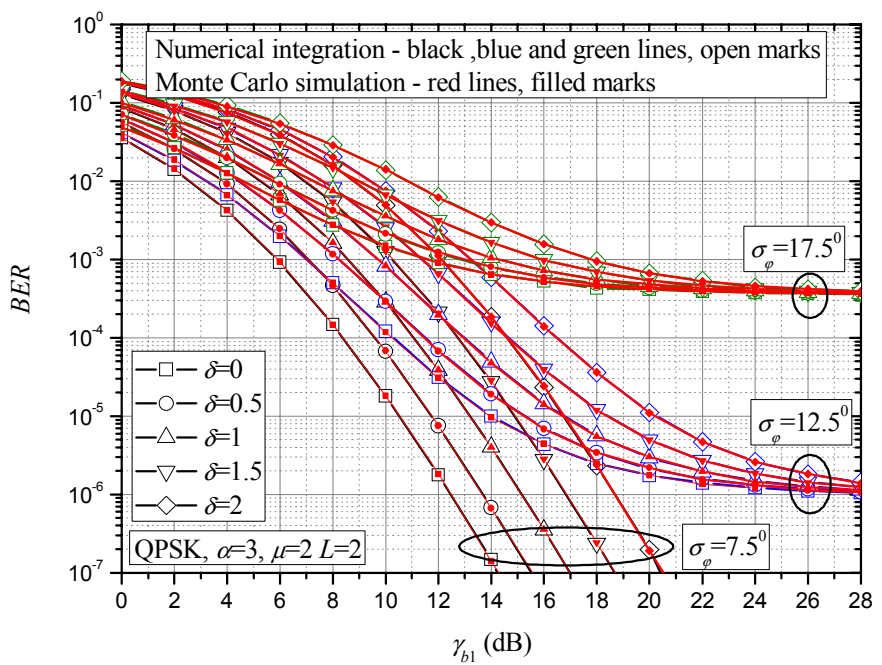

Fig. 4. BER of uncoded QPSK as a function of the average SNR per bit of the first input branch for different values of decaying power delay factor

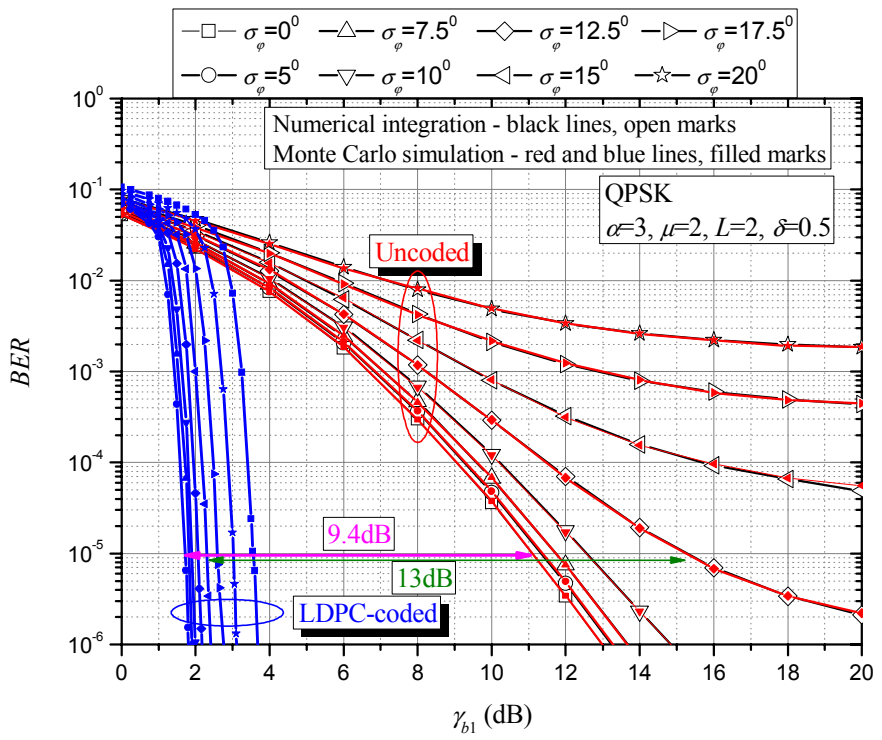

Fig. 5. BER of uncoded and LDPC-coded QPSK as a function of the average SNR per information bit of the first input branch
Fig. 6 illustrates the influence of diversity order on BER values for both uncoded and LDPC-coded QPSK signals. The coding gain is greater for smaller number of receiving antennas. Without diversity the BER of $10^{-4}$ can not be achieved without encoding because the BER floor about $2.5 \cdot 10^{-4}$ appears, but with LDPC code application even the lower BER of $10^{-6}$ is achieved for $\gamma_{b 1}=5 \mathrm{~dB}$. Similarly, in the case of dual branch diversity, the BER floor slightly greater than $10^{-6}$ appears for uncoded signals, while with LDPC coding this BER is achieved for $\gamma_{b 1}=1.4 \mathrm{~dB}$. Table I presents the numerical values illustrating the influence of diversity order on BER performance of uncoded and LDPC-coded EGC receiver.

Fig. 7 compares the simulation results of the proposed LDPC code and standard convolutional code $\mathrm{CC}(2,1,7)$ with code rate $R=1 / 2$, constraint length $K=7$, generating polynomial [171,133], and soft decision Viterbi decoding with decoding depth equal to 64. Our LDPC code with higher code rate $(R=0.75)$ performs better than $\mathrm{CC}(2,1,7)$. For example, at a BER of $10^{-6}$, the LDPC code outperforms convolutional code by about $1.9 \mathrm{~dB}$ for all values of parameter $\alpha$.

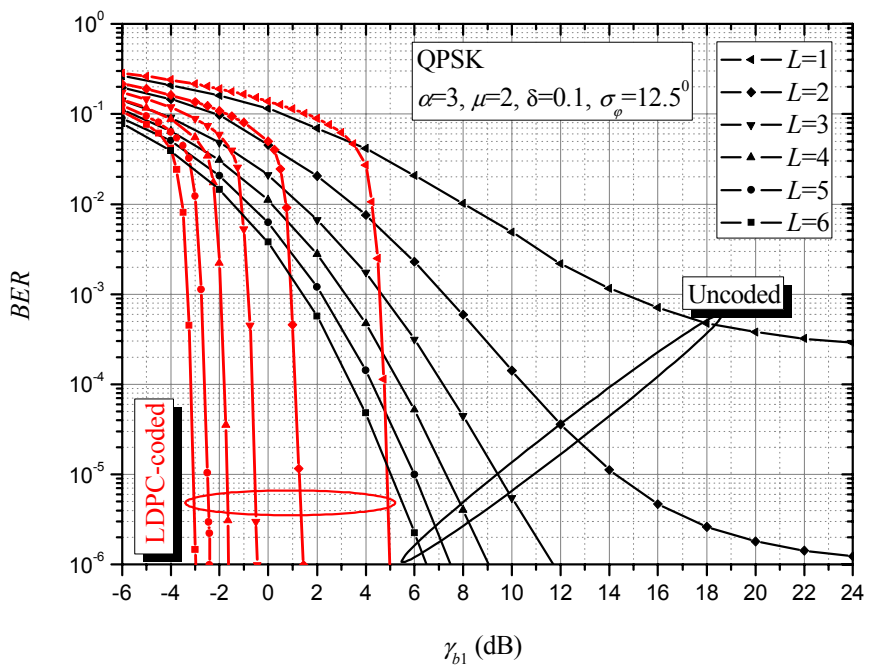

Fig. 6. BER of uncoded and LDPC-coded QPSK as a function of the average SNR per information bit of the first input branch for different diversity orders 


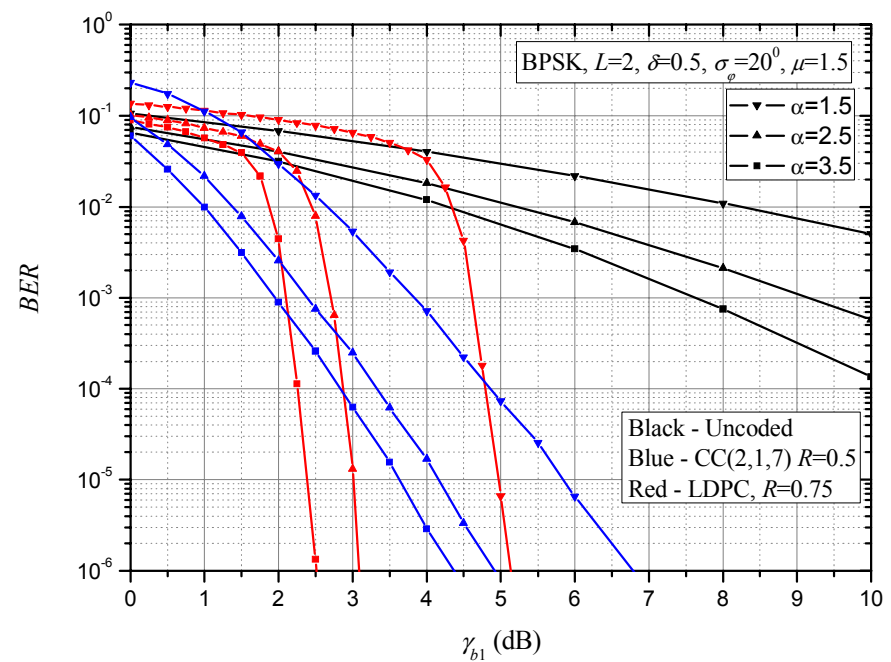

Fig. 7. BER of uncoded and coded BPSK as a function of the average SNR per information bit of the first input branch for different values of fading severity

TABLE I REQUIRED $\gamma_{b 1}$ VALUES (IN DECIBELS) IN ORDER TO ACHIEVE SPECIFIED BER VALUES FOR DIFFERENT DIVERSITY ORDERS

\begin{tabular}{|c|c|c|c|c|c|c|}
\hline \multirow{2}{*}{$\begin{array}{l}\text { BER } \\
L\end{array}$} & \multicolumn{2}{|c|}{$10^{-4}$} & \multicolumn{2}{c|}{$10^{-5}$} & \multicolumn{2}{c|}{$10^{-6}$} \\
\cline { 2 - 7 } & uncoded & coded & uncoded & coded & uncoded & coded \\
\hline 1 & & 4.8 & & 4.9 & & 5.0 \\
\hline 2 & 10.5 & 1.1 & 14.3 & 1.3 & & 1.4 \\
\hline 3 & 7.2 & -0.7 & 9.4 & -0.6 & 11.7 & -0.4 \\
\hline 4 & 5.4 & -1.8 & 7.3 & -1.7 & 9.0 & -1.6 \\
\hline 5 & 4.3 & -2.6 & 6.0 & -2.5 & 7.5 & -2.4 \\
\hline 6 & 3.4 & -3.2 & 5.0 & -3.1 & 6.5 & -3.0 \\
\hline
\end{tabular}

\section{SUMMARY}

In this paper, we have studied the performance of uncoded and LDPC-coded EGC receiver operating over generalized fading channel assuming imperfect cophasing and receiver branch unbalance. We have presented the results showing how much the QPSK modulation format is more sensitive to the phase error than the BPSK modulation format in the observed scenario. Also, we have emphasized the range of SNR values when receiver unbalance parameter value has considerable influence on BER performance and showed that receiver unbalance parameter does not influence the BER floor value. The regular LDPC code with moderate length and high code rate $(R=0.75)$ suitable for communication over this type system using PSK signaling has been designed using the concept of quasi-cyclic LDPC codes of large girth. The results have shown that this LDPC code provides significant coding gain with respect to an uncoded system in generalized fading channel and also is very tolerant to imperfect cophasing. The proposed LDPC code with code rate 0.75 outperforms the convolutional code with code rate 0.5 under previously mentioned influences. The determined results enable us to make compromise between the quality of the signal transmission (BER values) and the system complexity (the number of the receiver branches) for uncoded and LDPC coded signaling.

\section{ACKNOWLEDGEMENT}

This paper was supported in part by the NSF under Grant IHCS-0725405 and in part by the Ministry of Science of Serbia under Grant TR-11030.

\section{REFERENCES}

[1] M. K. Simon, M. S. Alouini, Digital Communication over Fading Channels, $2^{\text {nd }}$ ed. New York: Wiley, 2005.

[2] N. C. Beaulieu, A. A. Abu-Dayya, "Analysis of equal gain diversity on Nakagami fading channels," IEEE Trans. Commun., vol. 39, no. 2, pp. 225-234, Feb. 1991

[3] D. A. Zogas, G. K. Karagiannidis, S. A. Kotsopoulos, "Equal gain combining over Nakagami- $n$ (Rice) and Nakagami- $q$ (Hoyt) generalized fading channels," IEEE Trans. Wireless Commun., vol. 4, no. 2, pp. 374379, March 2005.

[4] N. C. Sagias, G. K. Karagiannidis, P. T. Mathiopoulos, T. A. Tsiftsis, "On the performance analysis of equal-gain diversity receivers over generalized Gamma fading channels," IEEE Trans. Wireless Commun., vol. 5, no. 10, pp.2967-2975, Oct. 2006.

[5] T. Piboongungon, V. A. Aalo, C.-D. Iskander, "Average error rate of linear diversity reception schemes over generalized Gamma fading channels," in Proc. IEEE Southeastcon, pp. 265-270, Ft. Lauderdale, Fl, Apr. 2005.

[6] M. A. Najib, V. K. Prabhu, "Analysis of equal-gain diversity with partially coherent fading signals," IEEE Trans. Vehic. Techn., vol. 49, no. 3, pp. 783-791, May 2000.

[7] N. C. Sagias, G. K. Karagiannidis, "Effects of carrier phase error on EGC receivers in correlated Nakagami-m fading," IEEE Commun. Lett., vol. 9, no. 7, pp. 580-582, July 2005.

[8] M. D. Yacoub, "The $\alpha-\mu$ distribution: a general fading distribution," in Proc. IEEE Int. Symp. PIMRC, vol. 2, pp. 629-633, September 2002.

[9] M. D. Yacoub, "The $\alpha-\mu$ distribution: a physical fading model for the Stacy distribution," IEEE Trans. Vehic. Techn., vol. 56, no. 1, pp. 27-34, Jan. 2007.

[10] W. C. Lindsey, M. K. Simon, Telecommunication Systems Engineering, New Jersey: Prentice-Hall, 1972.

[11] I. S. Gradsteyn, I. M. Ryzhik, Table of Integrals, Series, and Products, $6^{\text {th }}$ ed., New York: Academic, 2000.

[12] M. P. C. Fossorier, "Quasi-cyclic low-density parity-check codes from circulant permutation matrices," IEEE Trans. Inform. Theory, vol. 50, no. 8, 1788-1793, Aug. 2004.

[13] I. B. Djordjevic, L. Xu, T. Wang, and M. Cvijetic, "Large girth lowdensity parity-check codes for long-haul high-speed optical communications," in Proc. OFC/NFOEC 2008, Paper no. JWA53, San Diego, CA, Feb. 24-28 2008.

[14] R. Tanner, "A recursive approach to low complexity codes," IEEE Trans.Inform. Theory, vol. 27, no. 5, pp. 533-547, Sept. 1981.

[15] H. Xiao-Yu, E. Eleftheriou, D.-M. Arnold, and A. Dholakia, "Efficient implementations of the sum-product algorithm for decoding of LDPC codes," in Proc. IEEE Globecom 2001, Vol. 2, pp. 1036-1036E, Nov. 2001.

[16] M. C. Jeruchim, P. Balaban, K. Sam Shanmugan, Simulation of Communication Systems - Modeling, Methodology, and Techniques, New York: Kluwer, 2000.

[17] W. H. Press, S. A. Teukolsky, W. T. Vetterling, B. P. Flannery, Numerical Recipes in $C, 2^{\text {nd }}$ Ed., USA: Cambridge University Press, 2002. 